Introduction Lymphogranuloma venereum (LGV) continues to be difficult to diagnose and can lead to significant sequelae. Since 2011, all rectal specimens testing positive for Chlamydia trachomatis were tested for LGV serovars, leading to a greater number of LGV cases (mean, 21 cases/year for 2011-2014). In 2015, case reports of LGV doubled to 42 cases. We sought to characterise LGV cases reported in BC since 2011, and assess possible reasons for the 2015 increase.

Methods Demographic and behavioural information about all LGV cases reported in BC from January 1, 2011 to December 31, 2015 were identified. Provincial laboratory data were reviewed for potentially missed cases. LGV cases were categorised by reporting year (i.e., 2011-2014 and 2015) and analysed using the chi-square test or Fisher's exact test. LGV percent positivity was calculated as the number of LGV cases over the number of positive rectal chlamydia.

Results From 2011-2014, 83 cases were reported versus 42 in 2015. All were among men who have sex with men (MSM). The median age for cases was 46 years and 44 years for 2011-2014 and 2015, respectively $(\mathrm{p}=0.26)$. HIV co-infection was similar in both periods $(54 / 83$ in $2011-2014,25 / 42$ in 2015, $\mathrm{p}=0.61$ ). Of those known to be co-infected with HIV, the majority had undetectable viral loads (34/54 in 2011-2014 and $18 / 25$ in 2015). There was a decrease in the proportion of cases who identified as Caucasian from 2011-2014 to $2015(\mathrm{p}=0.004)$ and an increase in proportion of asymptomatic cases, although not statistically significant $(p=0.06)$. Percent positivity was $7.1 \%$ and $7.2 \%$ in $2011-2014$ and 2015 , respectively.

Conclusion The similar case characteristics and percent positivity during both periods, and increase in proportion of asymptomatic cases, suggest that increased screening for rectal sexually transmitted infections may be the reason for the observed increase in LGV cases. Further evaluation is needed to understand LGV trends, particularly among HIV-positive MSM who are disproportionately affected by LGV.

\section{P3.105 MATERNAL SYPHILIS IN BRITISH COLUMBIA, CANADA: 2010 TO 2016}

${ }^{1,2} \mathrm{~J}$ Wong, ${ }^{1,2} \mathrm{C}$ Arkell, ${ }^{2} \mathrm{M}$ Durigon, ${ }^{1} \mathrm{~S}$ Makaroff, ${ }^{1} \mathrm{C}$ Montgomery, ${ }^{1} \mathrm{M}$ Morshed, ${ }^{2,3} \mathrm{D}$ Money, ${ }^{4} \mathrm{~J}$ van Schalkwyk, ${ }^{4} \mathrm{~A}$ King, ${ }^{5} \mathrm{M}$ Gilbert, ${ }^{1,2} \mathrm{~T}$ Grennan, ${ }^{1,2} \mathrm{G}$ Ogilvie. ${ }^{1} B C$ Centre for Disease Control, Canada; ${ }^{2}$ University of British Columbia, Canada; ${ }^{3} B C$ Centre for Disease Control Public Health Laboratory, Canada; ${ }^{4} B C$ Women's Hospital, Canada; ${ }^{5}$ Perinatal Services $B C$, Canada

\subsection{6/sextrans-2017-053264.340}

Introduction From 2010 to 2016, syphilis rates have tripled among women in British Columbia (BC), Canada. We sought to characterise maternal syphilis cases in BC to identify areas to strengthen syphilis prevention programming.

Methods Virtually all syphilis tests in BC are performed at the provincial laboratory. Positive tests are reviewed by centrallylocated expert clinicians who diagnose, stage, and recommend treatment. Demographic and treatment information of syphilis cases (primary, secondary, early and late latent) diagnosed in pregnant women (or within 90 days after delivery) from January 2010 to July 2016 were reviewed and descriptive analyses performed. We assessed prenatal syphilis screening based on the prenatal flag on the laboratory requisition and compared against the number of live births reported by BC Vital Statistics.

Results From 2010 to 2015, 283168 syphilis tests were done as part of prenatal testing, compared with 264496 live births. From 2010 to July 2016, there were 45 maternal syphilis cases reported (18 early latent, 27 late latent-of note, syphilis screening by EIA commenced July 2014). The majority of cases (38/45) lived in Greater Vancouver; median age 30 years (range: 20-46). 27, 13, and 3 cases were diagnosed in the first, second, and third trimester, respectively; 2 were diagnosed post-partum. Treatment information was available on $44 / 45$ cases: 42 cases received $\geq 2$ penicillin injections and 2 received doxycycline. Being born outside Canada or having a partner in a developing country was the most common risk factor identified $(n=13)$. One case reported sex trade work, 4 reported having casual sex ( $>4$ partners), and 4 reported substance use. Few cases (6/34) reported $\geq 2$ partners in the last year.

Conclusion Most maternal syphilis cases are diagnosed by first trimester prenatal screening, but a few remain diagnosed postpartum. Increasing efforts to engage early for those born in high syphilis incidence countries (or whose partners remain in such countries) and repeat screening may be areas for focus.

\section{P3.106 UPTAKE OF HOME-BASED POINT-OF-CARE SYPHILIS \& HIV TESTING AMONG MALE PARTNERS OF PREGNANT WOMEN IN WESTERN KENYA}

${ }^{1}$ Jennifer Mark, ${ }^{2} \mathrm{~J}$ Kinuthia, ${ }^{1} \mathrm{~A}$ Roxby, ${ }^{1} \mathrm{D}$ Krakowiak, ${ }^{3} \mathrm{~A}$ Osoti, ${ }^{4} \mathrm{~B}$ Richardson, ${ }^{2}$ Gone Ma, ${ }^{2} \mathrm{~V}$ Asila, ${ }^{1} \mathrm{~S}$ Parikh, ${ }^{1} \mathrm{C}$ Farquhar. ${ }^{1}$ University of Washington, Seattle, USA; ${ }^{2}$ Kenyatta National Hospital, Nairobi, Kenya; ${ }^{3}$ University of Nairobi, Nairobi, Kenya; ${ }^{4}$ Fred Hutchinson Cancer Research Centre, Seattle, USA

\subsection{6/sextrans-2017-053264.341}

Introduction Few men are tested for syphilis or HIV during their partners' pregnancy, a period of high HIV transmission risk and preventable adverse pregnancy outcomes. Offering home-based STI education and point-of-care (POC) testing of syphilis to couples can support HIV programs to reduce transmission of sexually transmitted infections and adverse pregnancy outcomes.

Methods We assessed male partner uptake of paired POC syphilis and HIV tests within a randomised controlled trial (RCT) of 600 pregnant women and their male partners in Kenya. Married or cohabiting women were unaccompanied and attending a clinic-based first antenatal visit at recruitment. Participating men received a couple home-based visit with testing during pregnancy or at 6 months postpartum. We also evaluated whether the addition of syphilis testing has an effect on the uptake of HIV testing among men.

Results From September 2013 to June 2014, male participation in home-based visits was $85 \%$ among women remaining enrolled (260 during pregnancy, 240 postpartum). Paired testing was offered to subsets of 80 and 230 men during pregnancy and postpartum. In both groups, test uptake was high: 1) For syphilis, $91 \%$ men agreed to test during pregnancy and 96\% agreed postpartum; 2) For paired syphilis and HIV testing, 91\% of men tested for both during pregnancy and $98 \%$ tested postpartum. Third, adding syphilis testing did not 